\title{
T-RANS Simulations of Subcritical Flow with Heat Transfer Past a Circular Cylinder Surrounded by a Thin Porous Layer
}

\author{
M. P. Sobera • C. R. Kleijn
}

Received: 3 October 2006 / Accepted: 18 March 2008 /

Published online: 23 April 2008

(C) The Author(s) 2008

\begin{abstract}
We study flow and heat transfer to a cylinder in cross flow at $R e=$ 3,900-80,000 by means of three-dimensional transient RANS (T-RANS) simulations, employing an RNG $k-\epsilon$ turbulence model. Both the case of a bare solid cylinder and that of a solid cylinder surrounded at some fixed distance by a thin porous layer have been studied. The latter configuration is a standard test geometry for measuring the insulating and protective performance of garments. In this geometry, the flow in the space between the solid cylinder and the porous layer is laminar but periodic, whereas the outer flow is transitional and characterized by vortex shedding in the wake of the cylinder. The results from the T-RANS simulations are validated against data from Direct Numerical Simulations and experiments. It is found that T-RANS is very well suited for simulating this type of flow. The transient nature of the flow underneath the porous layer is well reproduced, as well as the influence of vortex shedding on the heat transfer in the downstream stagnation zone. T-RANS results are found to be in much better agreement with DNS and experimental data than results from steady-state RANS.
\end{abstract}

Keywords T-RANS • Circular cylinder $\cdot$ Porous medium $\cdot$ CFD

\section{Introduction}

The study of insulating and protective performance of (e.g. NBC protective) garments involves aspects from a gamut of disciplines such as physiology, biology, chemistry, physics, mechanics, heat and mass transfer and fluid dynamics. These phenomena take place at a wide range of scales, from the molecular scale of chemical reactions, via the millimeter scales of e.g. textile fibers, to the meter scale of a full

M. P. Sobera · C. R. Kleijn (凶)

Department of Multi-Scale Physics, Delft University of Technology,

Prins Bernhardlaan 6, 2628BW, Delft, The Netherlands

e-mail: C.R.Kleijn@TUDelft.NL 
human body. At the latter scale, fluid dynamics, turbulence and heat and mass transfer play a crucial role. Their study is complicated by the complex geometry, interactions between the fluid flow and the moving garments and body, and the interactions between different scales.

In order to reduce the complexity of the problem and to introduce some standardization, a simplified set-up has been introduced to test garments [1]. Rather than a full clothed and moving human body, it consists of a solid cylinder-representing a limb-surrounded at some small but fixed distance by a layer of (porous) garment material. This is schematically depicted in Fig. 1. This setup is widely used to determine e.g. chemical protection and hydraulic properties of protective garments, as well as breakthrough characteristics for poisonous species and aerosols deposition. With typical air velocities of $1-10 \mathrm{~m} / \mathrm{s}$ and limb diameters of the order of $0.1 \mathrm{~m}$, the range of relevant Reynolds numbers is 5,000-50,000.

Apart from being a standard setup for experimental research, the configuration described above has also been the starting point for many modeling studies [1-3]. In these models, the dynamics of the air flow underneath the garment is usually highly simplified, e.g. neglecting temporal and spatial variations. As a result, these models predict averaged (in time and space) characteristics of the garments only. In practice, however, the interest is in local and instantaneous properties in order to identify the most critical spots. For this, information is needed on local and time dependent flow characteristics underneath the garment, which are obviously determined by the transient and turbulent flow around it.

However, in contrast to the well-known case of flow around a solid circular cylinder, the above configuration, which is also of practical relevance for instance in filtering and separation technology, has received little attention. In literature Watanabe and coworkers have reported an experimental study of the velocity field around a fabric-covered cylinder [4]. They studied the influence of the porous layer permeability on the velocity of the flow underneath the porous layer. Experimental results on heat transfer to a fabric covered cylinder have been reported by Kind and coworkers [5]. Gibson [6] presented numerical computations of the air flow and heat transfer to a fabric-covered cylinder. A numerical study of free and mixed convection around a cylinder surrounded by porous material, focusing on situations where there is no or very little external air flow, was presented by Bo and coworkers [7].

Fig. 1 Computational domain and discretization

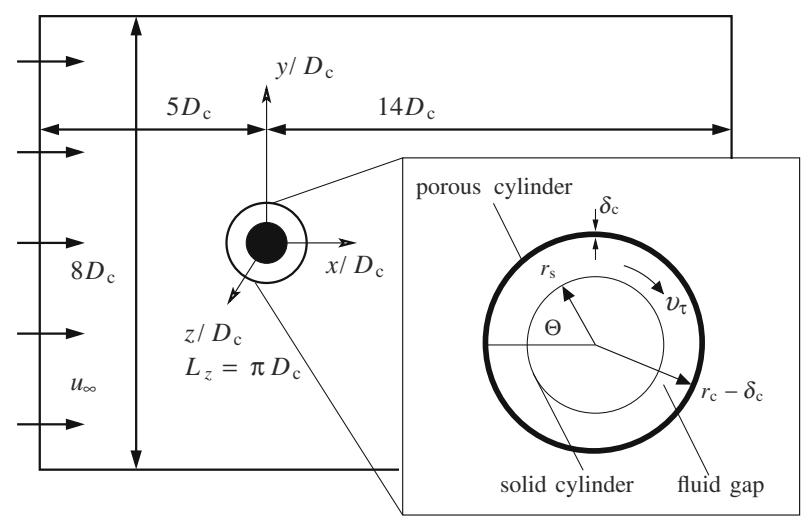


In our previous work [8], we reported CFD simulations of fluid flow, heat and mass transfer for the geometry of Fig. 1 by means of the Reynolds averaged Navier-Stokes (RANS) approach. From these simulations, we deduced scaling rules for the flow characteristics as a function of the outer flow Reynolds number, the Darcy number, which is a dimensionless measure for the hydraulic permeability of the porous layer of garment material, and geometric ratios. The RANS approach, however, raised several questions regarding the assumed laminar and steady nature of the flow underneath the porous layer. Therefore, in a more recent publication [9], we used Direct Numerical Simulations to study the precise nature of the flow underneath the porous layer, for a subcritical outer flow. The flow underneath the porous layer was found to be laminar and periodic in character, with a frequency locked to the vortex shedding frequency of the outer flow. This periodicity, which cannot be accounted for by RANS simulations, is expected to have an influence on the heat and mass transfer to the solid cylinder. The DNS approach, on the other hand, is computationally very expensive and limited to relatively low Reynolds numbers: in our DNS study [9], the outer flow Reynolds number was $R e=3,900$, which is at the very low end of the range of interest for testing garment properties.

For practical application in realistic studies of garment properties, we are in need of a CFD approach which, on the one hand, satisfactorily accounts for the periodic nature of the flow around and underneath the porous layer, and on the other hand is suitable for application to high Reynolds number flows and in complex geometries, such as around a full, moving human body. Moreover, the approach should allow for ultimately including modeling aspects related to e.g. vapor transport and condensation, chemical reactions, fluid-structure interactions and moving boundaries, and should be suitable for application by specialists from different disciplines. Therefore, it is preferred that the CFD approach would be available within the context of a general purpose CFD code.

The modeling issues critical for this type of flow are similar to those for modeling the flow around blunt bodies, including strongly unsteady and transitional flow behavior even at low and moderate $\operatorname{Re}[10,11]$. A first obvious option would therefore be to use large eddy simulations (LES). For these types of flows, LES are more accurate than any RANS approach. However, LES has serious limitations in accurately predicting wall heat transfer and friction in complex geometries and at realistically high Reynolds numbers [12]. This is due to the high demands on nearwall grid resolution, not only in the wall-normal direction, but also in the streamwise and span-wise directions in order to properly resolve the small eddies and streaky structures characteristic for the near-wall region. A possible alternative is the so-called time-dependent Reynolds averaged Navier-Stokes (T-RANS) method. This approach combines traditional LES with single-point RANS turbulence closure for unresolved scales [13]. The basic rationale is to resolve only large coherent or deterministic structures by direct solution of the Navier-Stokes equations, whereas small-scales are modeled. Modeling a larger part of the spectrum (as compared to LES) requires a more sophisticated model than the standard sub-grid scale model for LES. For this, a form of RANS model is applied that is not related to the size of the numerical mesh. In comparison with conventional RANS, in the T-RANS approach the subscale model covers only the incoherent or random fluctuations, whereas the large-scales are fully resolved. As compared to LES, in which most of the contributions to the long-term statistical averages are fully resolved, in T-RANS 
both the resolved and unresolved contributions are of the equal order of magnitude. This method, which has been used and tested for various applications, e.g. [14-19], assumes that the characteristic time scale of the coherent motion is sufficiently large compared to the time scale of the turbulent fluctuations. In our earlier DNS study [9], we indeed found that for the flow under consideration the time scale of vortex shedding is significantly larger than the characteristic time scale of the turbulent fluctuations. The intrinsic transient nature (as opposed to RANS methods) and the required relatively course temporal and spatial discretization (as compared to LES and DNS) make the T-RANS approach very promising for our final goal, i.e. the accurate modeling of flow, heat transfer around a full, moving human body.

The main goal of this study is to investigate the performance and accuracy of the T-RANS approach for modeling the flow, heat transfer in the described testgeometry of Fig. 1. Because of reasons mentioned above, the study was performed using a general purpose CFD code, viz. Fluent 6.2. In an earlier study [9], we found that DNS simulations with Fluent for the same geometrical configuration as studied here did compare very well to those obtained with an in-house code and with DNS data from literature. The present Fluent T-RANS results are validated against our earlier DNS results for $R e=3,900$ [26] and against laser doppler anemometry experimental data. After validation, the T-RANS approach is applied to higher Reynolds number flows and is used to study the scaling behavior of the flow. Results are compared to the scaling rule based on RANS simulations which we presented earlier [8].

\section{Numerical Approach}

\subsection{Computational domain}

The computational domain we used here is schematically depicted in Fig. 1. It is identical to the one used in our earlier DNS study [9]. The domain extends from $x=-5 D_{\mathrm{c}}$ to $x=14 D_{\mathrm{c}}$ in the streamwise direction, from $y=-4 D_{\mathrm{c}}$ to $y=4 D_{\mathrm{c}}$ in the normalwise direction, and from $z=-\pi D_{\mathrm{c}} / 2$ to $z=\pi D_{\mathrm{c}} / 2$ in the spanwise direction, where $D_{\mathrm{c}}=2 r_{\mathrm{c}}$ is the outer cylinder diameter. The dimensionless thickness of the fluid gap (the distance between the solid cylinder surface and the porous layer) is $I g=\delta_{\mathrm{g}} / D_{\mathrm{c}}=5 \times 10^{-2}$ and the dimensionless thickness of the porous layer is $I c=$ $\delta_{\mathrm{c}} / D_{\mathrm{c}}=5 \times 10^{-3}$.

Although the time averaged flow and temperature field are expected to be twodimensional, due to the use of periodic boundary conditions in the spanwise (and normalwise) direction, we performed three-dimensional simulations. The reason for this is that we are interested in a computational approach that does not only correctly predict the time-averaged flow and heat transfer, but rather the local and temporal extremes. It is especially these extremes which determine the protective qualities of the garment. In addition it is noted here that attempts to perform two-dimensional T-RANS for the flow around a solid cylinder led to inaccurate solutions, with a strongly under-estimated recirculation length.

For grid generation we used the commercial pre-processor Gambit 2.2. The three-dimensional unstructured grid consisted of 500,000 (hexagonal) grid cells (as compared to six million cells in our earlier DNS study [9]). As in the DNS study, 
local grid refinement was applied in the vicinity of the cylinder and in the near wake region. In this region typical grid sizes were $\Delta x=\Delta y=0.03 D_{\mathrm{c}}$. Further from the cylinder the grid size, increased to $\Delta x=\Delta y=0.2 D_{\mathrm{c}}$. The spanwise grid size was fixed and equal to $\Delta_{z}=0.157 D_{\mathrm{c}}$ in the entire domain.

A uniform velocity $u_{\infty}$ and temperature $T_{\infty}$ have been specified at the inlet at $x / D_{\mathrm{c}}=-5$. At the surface of the inner cylinder, a constant temperature and noslip boundary condition have been imposed. Based on our earlier DNS results, we assumed the flow inside the air gap to be laminar. Therefore, we did not apply wall functions at the solid cylinder surface. The porous sheath was treated as a fluid zone, where a pressure drop was imposed (see below).

The solution was performed by means of the CFD package Fluent 6.2. For spatial discretization we employed the QUICK [20] scheme and in time we applied a second order implicit scheme. The time step size was $\Delta t=0.1 D_{\mathrm{c}} / u_{\infty}$, as compared to $\Delta t=$ $0.001 D_{\mathrm{c}} / u_{\infty}$ in our earlier DNS study. All of the simulations were performed by means of a parallel solver, running on an in-house Linux based PC cluster, typically using eight processors on four double-CPU computational nodes.

\subsection{Porous layer model}

Inside the porous zone, the momentum equation was extended with an additional source term. When the Reynolds number based on typical velocities and structural dimensions in the porous layer is small, the momentum source term in the porous zone follows from Darcy's law:

$$
\mathbf{v}=-\frac{\overline{\bar{k}}}{\mu} \cdot \nabla P
$$

where $\overline{\bar{k}}$ is the permeability tensor, $\mathbf{v}$ is the superficial velocity through the porous medium and $\mu$ is the fluid viscosity. Assuming a scalar permeability $k$ in the direction of the flow velocity $v$, the above means that total pressure drop over a thin porous layer of the thickness $\delta_{\mathrm{c}}$ equals:

$$
\Delta P=-\frac{\mu}{k} v \delta_{\mathrm{c}}
$$

Rewriting the above relation in dimensionless form (for details see [8]), we obtain:

$$
\Delta \widehat{P}=-\frac{\widehat{v}}{\operatorname{ReDaIc}}
$$

with $\widehat{v}$ the air velocity scaled with the free stream velocity $u_{\infty}, \Delta \widehat{P}$ the dimensionless pressure drop over the porous zone scaled with the free stream dynamic pressure $\rho u_{\infty}^{2}, R e$ free stream Reynolds number $\rho u_{\infty} D_{\mathrm{c}} / \mu$ and $D a=k / \delta_{\mathrm{c}}^{2}$ the dimensionless permeability. In our earlier RANS study, the denominator of eq. (3) was found to play an important role in the scaling behavior of the flow.

Since we treated the porous layer as a fluid zone, no boundary conditions had to be imposed on the inner and outer interfaces of the porous layer. Values of practical interest have been used for the hydraulic permeability $k\left(10^{-11}-10^{-9} \mathrm{~m}^{2}\right)$ of the porous layer and its thickness $\delta_{\mathrm{c}}\left(10^{-3} \mathrm{~m}\right)$, leading to Darcy numbers $D a=k / \delta_{\mathrm{c}}^{2}$ in the range $10^{-5}-10^{-3}$. 


\subsection{Turbulence model}

The flow of interest in the present study is in the subcritical flow regime and strongly unsteady. This means that the use of RANS turbulence modeling is difficult and certainly not fully appropriate throughout the computational domain. The boundary layer at the (outer) cylinder surface, for instance, is fully laminar up to the separation point and the transition to turbulence occurs in the shear layer or in the wake.

In steady RANS approaches, the standard $k-\epsilon$ turbulence model has known weaknesses in predicting flows such as the one of interest in this work. In the present study, we were also unable to reproduce the presence of the vortex shedding phenomena in the flow by means of the standard $k-\epsilon$ model in combination with a T-RANS approach. Therefore, we used its variant proposed by Yakhot and Orszag [21-23], the RNG $k-\epsilon$ model. It is derived from the instantaneous Navier-Stokes equations by using a mathematical technique called renormalization group theory. Velayati and Yaghoubi [24] reported that the RNG based $k-\epsilon$ is more responsive to the effects of flow separation, reattachment and recirculation than the standard $k-\epsilon$ model. Casey and Wintergerste in [25], recommend the use of RNG $k-\epsilon$ for low $R e$ flows.

\section{Results and Discussion}

\subsection{Validation}

\subsubsection{Flow and heat transfer around a solid cylinder at $R e=3,900$}

To validate our modeling approach, we first performed a validation study in which we compared Fluent T-RANS simulations of the flow and heat transfer around a solid cylinder at $R e=3,900$ against data from our earlier DNS study and against experimental and DNS data from literature. Please note, that for the geometry presented in Fig. 1 we now have $D_{\mathrm{c}}=2 r_{\mathrm{c}}=2 r_{\mathrm{s}}$ and $\delta_{\mathrm{c}}=0$.

Figure 2 presents a comparison of the time-averaged longitudinal velocity $v_{x}$ behind the cylinder at $(y=z=0)$, as a function of the dimensionless downstream $x$ position. The present T-RANS results are compared to our earlier DNS results [26], to DNS results of Tremblay [27], and to various experimental data from literature. The overall agreement is very good. The predicted length of the recirculation bubble (where $v_{x}<0$ ) is slightly shorter than in both DNS data sets, which might be an effect of the dissipative nature of the applied QUICK spatial discretization scheme. Breuer [30], in his LES study, reported shortened recirculation lengths when differencing schemes were employed that were more dissipative than central differencing schemes. He showed that in LES the influence of the discretization scheme on the recirculation length is in fact much stronger than that of the sub-grid scale model. In the present approach, an extra dissipation source is introduced via the turbulence model, which may add to an additional shortening of the recirculation bubble. However, the recirculation bubble length as predicted by T-RANS is in much better agreement with DNS data than our earlier RANS results, and also the agreement with experimental data is quite good. Similarly to our DNS results, the maximum velocity magnitude in the recirculation zone is slightly over predicted, 
Fig. 2 Time averaged streamwise ( $=x$-direction) velocity along the centerline $(y=z=0)$ at $\operatorname{Re}=3,900$

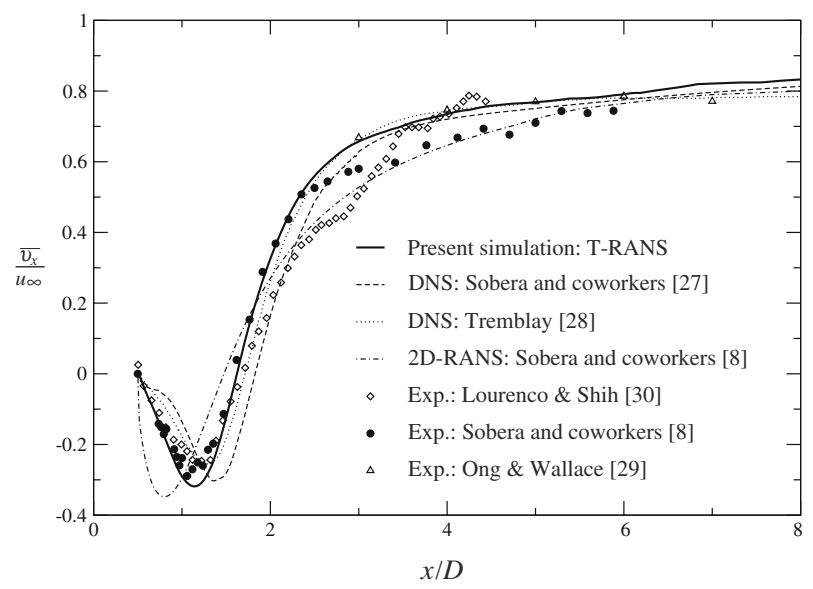

which is ascribed to the size of the computational domain in the transverse $(y-)$ direction, which was only $8 D_{\mathrm{c}}$ and considerably smaller than the $20 D_{\mathrm{c}}$ used by Tremblay.

In Fig. 3 the time history of the transverse velocity $v_{y}$ component in a point located in the wake at $\left(x=3 D_{\mathrm{c}}\right.$ and $\left.y=z=0\right)$ is presented. The results from our present simulation are compared to our previous DNS data reported in [9]. The agreement between the T-RANS approach and DNS is excellent. The dimensionless vortex shedding frequency $\left(S t=f D_{\mathrm{c}} / u_{\infty}\right)$ is $S t=0.205$ and $S t=0.214$ for present T-RANS and DNS, respectively.

Finally, we compared T-RANS predictions of the heat transfer to the solid cylinder surface to those of two-dimensional steady RANS simulations, employing the RNG $k-\epsilon$ model with wall functions, and to available experimental data from literature. No DNS data are available for the heat transfer. In Fig. 4 the timeaveraged Nusselt number as a function of the angular coordinate is presented. The angular positions $\Theta=0$ and $\Theta=180^{\circ}$ correspond to the front and back stagnation point, respectively. To facilitate comparison of the hydrodynamics to many published studies, the T-RANS simulations were performed at $R e=3,900$. No experimental data on heat transfer are available for this precise value of $R e$. We therefore used

Fig. 3 Comparison of the time history of the transverse velocity component obtained by means of DNS (solid line) and T-RANS (dashed line) in the wake behind the cylinder $\left(x / D_{\mathrm{c}}=3, y / D_{\mathrm{c}}=z / D_{\mathrm{c}}=\right.$ $0), \operatorname{Re}=3,900$

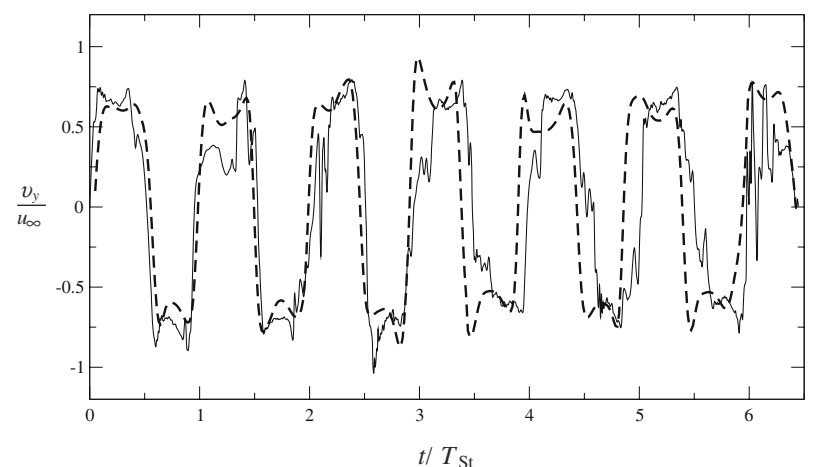


Fig. 4 Time averaged Nusselt number as a function of angle $\Theta$ computed for a solid circular cylinder

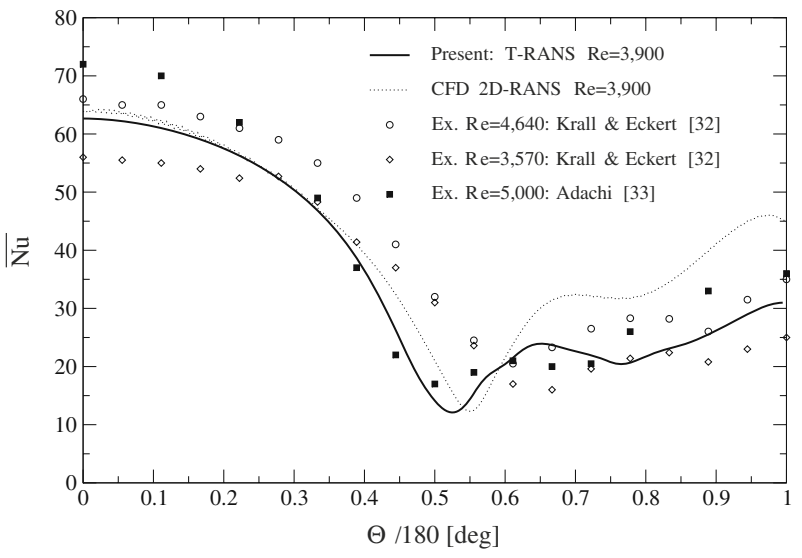

the closest experimental data from Krall and Eckert [31] at $R e=3,570$ and $R e=$ 4,640, as well as the closest results of Adachi and coworkers [32] at $R e=5,000$ for comparison. As in the T-RANS simulations, all experimental data were obtained for air as flowing medium. The overall quantitative agreement between T-RANS predicted and measured local Nusselt numbers is very good, particularly in the front and back stagnation point areas. In the back stagnation point area, where the heat transfer is most strongly influenced by the periodic vortex shedding, the T-RANS results are in significantly better agreement with experimental data than the RANS results. Some discrepancy is observed as to the location of minimum heat transfer. Krall and Eckert find this minimum around $\Theta=120^{\circ}$, whereas both in the T-RANS simulations and in Adachi's experimental data the minimal value of $\mathrm{Nu}$ occurs around $\Theta=90^{\circ}$.

\subsubsection{Flow and heat transfer around a covered cylinder}

To further validate the T-RANS approach performance for the geometry of particular interest to the present study as depicted in Fig. 1, we again compared present T-RANS results to those of our earlier DNS study [9], but now for a cylinder covered by a porous layer, at $R e=3,900, D a=2 \cdot 10^{-3}$ and $I c=5 \cdot 10^{-3}$. In Fig. 5, the time records of the tangential velocity inside the air gap underneath the porous layer and of the transverse velocity in the wake behind the cylinder are presented jointly. Curves in the upper part of the graph come from the present T-RANS study, whereas in the lower part of the graph DNS data from our earlier study [9]) are presented. The inner flow oscillations are locked to the vortex shedding occurring on the outer surface of porous layer. The upper graph confirms that exactly the same low frequency oscillating flow pattern underneath the porous layer is found in T-RANS and in DNS. The agreement between the dimensionless amplitude and frequency for both velocity signals is very good.

In the next graph (Fig. 6) the time-averaged dimensionless tangential velocity component, measured along the air gap centerline, as a function of angle is presented. The time-averaged T-RANS results are compared to time-averaged data from our earlier DNS study. The maximum tangential velocity occurs at $\Theta=50^{\circ}$, for both data sets. The magnitude of the velocity maximum is slightly lower for the unsteady RANS 
Fig. 5 The time history of the tangential velocity component inside the fluid gap at $\Theta=45^{\circ}$ and $\left(r-r_{s}\right) /\left(r_{c}-r_{s}\right)=0.5$ (dashed line) and the transverse velocity component in the wake behind the obstacle at $x / D_{\mathrm{c}}=3$ and $y / D_{\mathrm{c}}=0$ (solid line). Results from present T-RANS simulations (upper graph) are compared to DNS data from [9] (bottom graph), $R e=3,900$

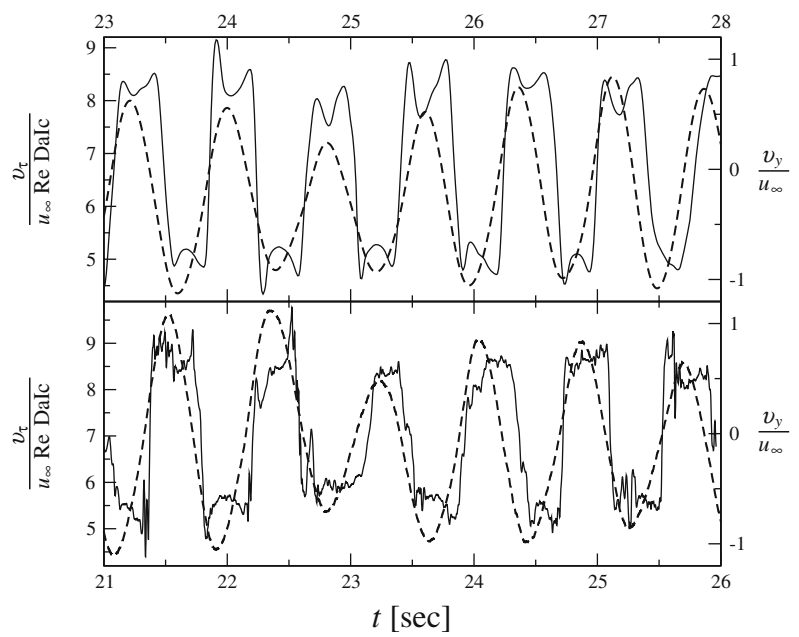

simulations as compared to the DNS simulations, whereas the velocities further downstream are somewhat over predicted compared to DNS. On average, however, the agreement between T-RANS and DNS is very good. Clearly, compared to the DNS results, the T-RANS results are in much better agreement than our earlier two-dimensional RANS simulations.

Finally, again for the case of a solid cylinder covered by a porous layer and a Reynolds number of $R e=3,900$, we compared the predicted time-averaged heat transfer to the solid cylinder surface. Figure 7 shows the time-averaged Nusselt number from the present T-RANS study, compared to that of our earlier DNS study and to that of our earlier two-dimensional RANS study, as a function of angular position on the surface. In the vicinity of the front stagnation point all three curves coincide, showing that the heat transfer predictions in this region are well reproduced even by means of two-dimensional steady state RANS. In the back part of the cylinder, for $\Theta>60^{\circ}$, however, the discrepancy between DNS/T-RANS results on the one hand, and two-dimensional RANS results on the other hand is significant.

Fig. 6 The dimensionless time averaged tangential velocity component measured along the fluid gap centerline as a function of angular position $\Theta$, $R e=3,900$

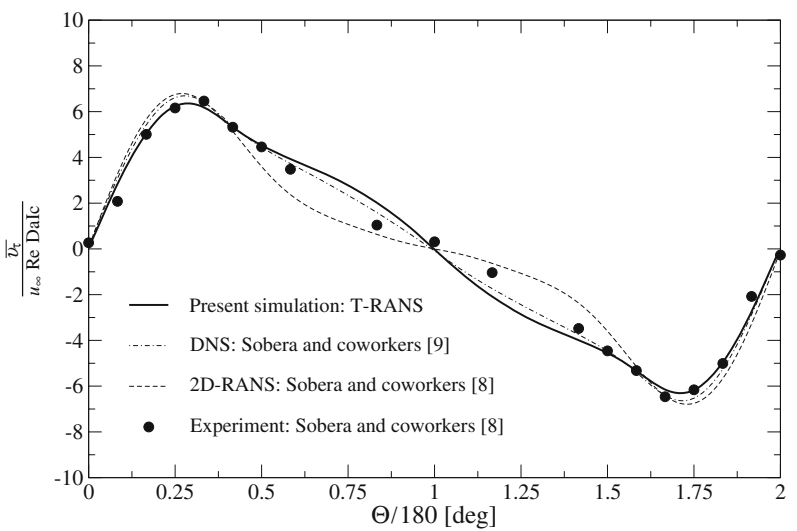


Fig. 7 Time averaged Nusselt number as a function of angle $\Theta$ computed for a circular cylinder surrounded by a porous layer, $R e=3,900$ $D a=2 \cdot 10^{-3}, I c=5 \cdot 10^{-3}$

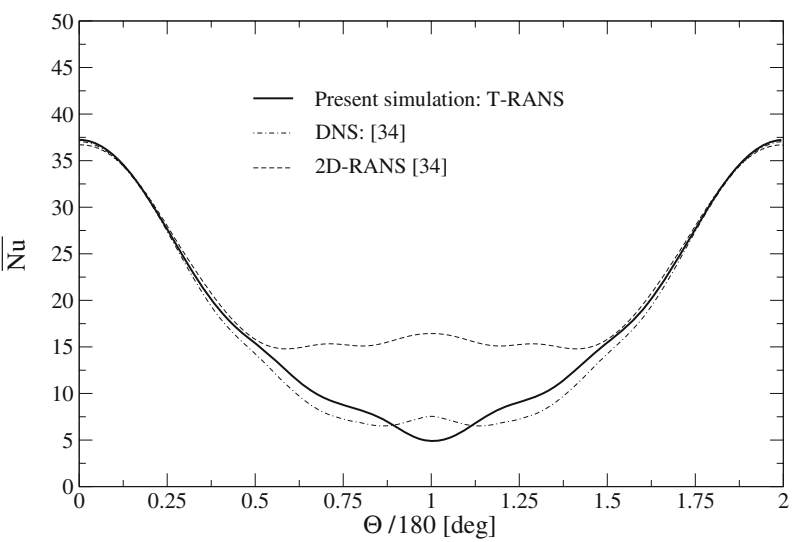

This is due to the transient nature of the flow, especially in the downstream region where there is a strong interaction with the shedding vortices. This was not captured by the two-dimensional RANS simulations, which were performed in steady state and for only one half of the cylinder, with a symmetry boundary condition at $\Theta=180^{\circ}$. Therefore, in the RANS simulations there was almost no flow underneath the porous layer in the downstream region. As a consequence, the heat transfer in this region was mainly conductive, with the thickness of the thermal boundary layer determined by the size $\left(r_{\mathrm{c}}-r_{\mathrm{s}}\right)$ of the air gap between the cylinder and the porous layer. This led to a Nusselt number $N u_{D_{\mathrm{c}}} \approx D_{\mathrm{c}} /\left(r_{\mathrm{c}}-r_{\mathrm{s}}\right)$. The present T-RANS
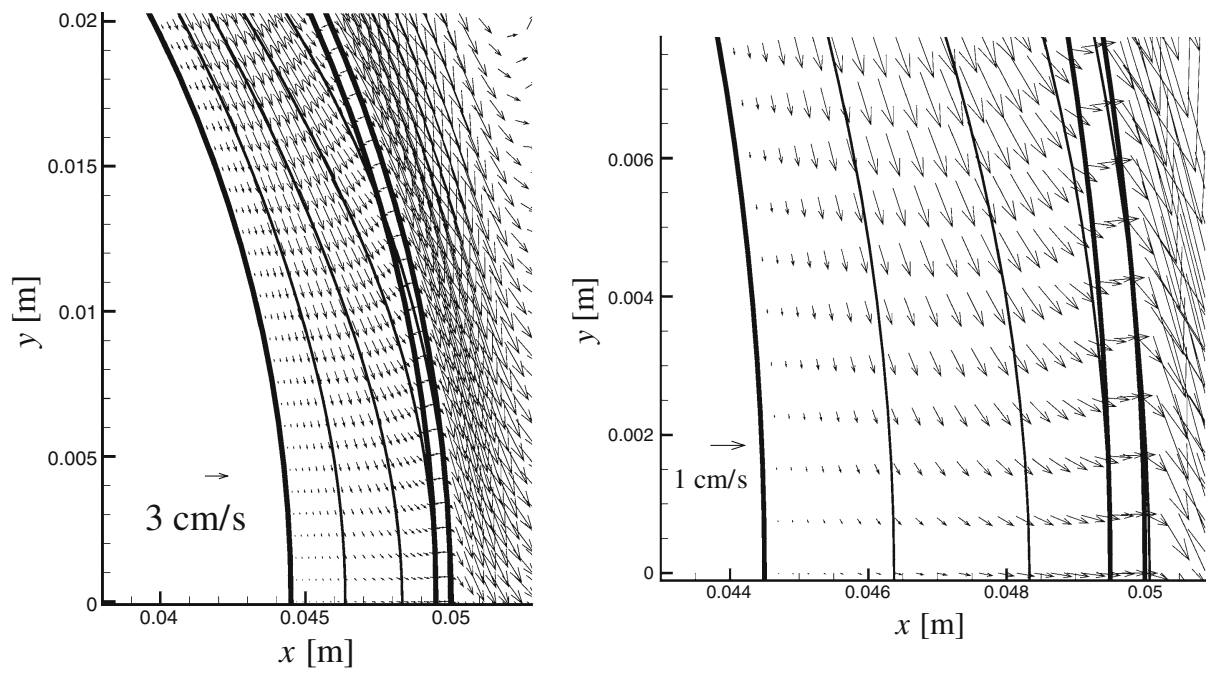

Fig. 8 Predicted time averaged velocity and temperature field in and around the air gap in the back stagnation point. The right-hand figure is zoomed in from the left-hand figure. The solid cylinder surface is at $r=0.0445 \mathrm{~m}$. The porous layer is between $r=0.0495 \mathrm{~m}$ and $r=0.05 \mathrm{~m}$. Isotherms have been drawn for dimensionless temperatures 0.9, 0.8. 0.7 and 0.6. Due to the radial flow component away from the solid surface, the isotherms are being pushed away from that surface 
Fig. 9 Time averaged normalised streamwise (=x-direction) velocity along the centerline $(y=z=0)$ for the covered cylinder situation of Fig. 8

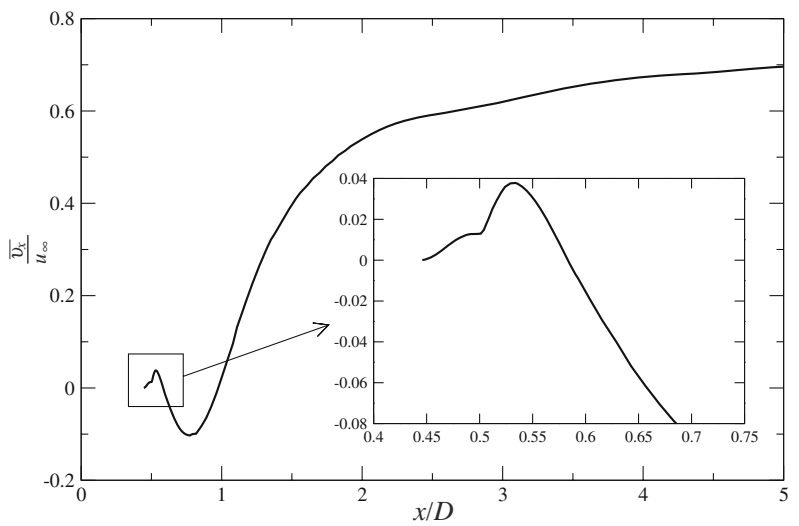

approach is much better capable of capturing the transient flow in the downstream region of the air gap. As shown in Fig. 8, this flow on average has a radial component away from the cylinder surface. For the case shown, these radial velocities are of the order of $5 \mathrm{~mm} / \mathrm{s}$, resulting in a radial Peclet number $u_{\mathrm{r}} \delta_{\mathrm{g}} / a_{\text {air }} \sim 1.2$. As a result, the thermal boundary layer is being pushed away from the cylinder surface, resulting in a decreased heat transfer. This was observed in both our earlier DNS and in the present T-RANS simulations. The agreement between DNS and T-RANS is quite satisfying.

It is interesting to note that, in contrast to the well-known case of an uncovered solid cylinder (Fig. 2), we observe positive (=away from the cylinder) $v_{x}$ velocities along the centerline. As shown in Fig. 9, this is a local effect occurring only in the close vicinity of the cylinder's back stagnation point. Further downstream, the negative velocities induced by the time mean recirculation take over, as in the case of a non-covered cylinder.

\subsection{Scaling rules}

In [8], we used two-dimensional steady state RANS simulations to study the scaling behavior of flow and heat transfer in the configuration from Fig. 1 as a function of the free stream Reynolds number, the dimensionless hydraulic permeability as expressed in the Darcy number, and the geometric ratios $I c=\delta_{\mathrm{c}} / D_{\mathrm{c}}$ and $I g=\left(r_{\mathrm{c}}-r_{\mathrm{s}}\right) / D_{c}$. From our earlier DNS simulations [9] and the above T-RANS simulations, however, it became clear that the RANS simulations did not adequately capture the transient nature of the flow in the air gap, resulting in significant errors in predicted velocity and heat transfer profiles and necessitating the re-evaluation of the scaling rules as obtained by RANS. DNS, however, is obviously not suitable for performing the necessary parameter variations, if only for the fact that it cannot possibly be applied to the entire range of Reynolds numbers of interest $(R e=4,000-80,000)$. Fortunately, as described above, we found T-RANS to be a good alternative. Unlike RANS, the T-RANS approach captures the important transient characteristics of the flow and heat transfer. And unlike DNS, it can be used in a parameter study. Therefore, in this section, we use T-RANS simulations to re-evaluate our earlier proposed, RANS based, scaling rules. 
Fig. 10 The dimensionless tangential velocity (averaged over time and angular coordinate) as a function of the scaling parameter $u_{\infty} \operatorname{Re} \mathrm{DaIc}$

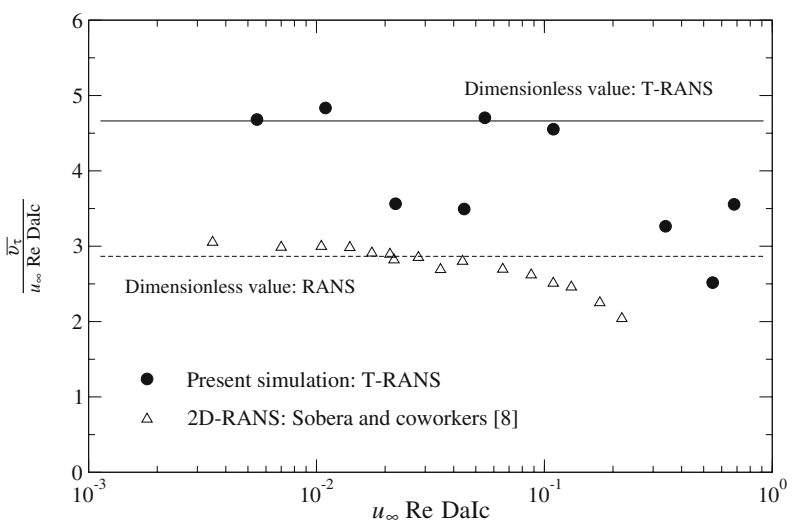

\subsubsection{The influence of the scaling parameter $u_{\infty}$ Re DaIc}

In our RANS study [8], we found that the tangential velocity underneath the porous layer approximately scales with $u_{\infty} R e$ DaIc over a range of more than 2 decades variation in that scaling parameter. This is shown in Fig. 10, where the so scaled tangential velocity component, averaged over time and angle, is presented. The scaling rule was deduced theoretically from a balance between the stagnation point pressure and the pressure drop over the porous layer, assuming frictionless flow in the air gap. In the two-dimensional RANS simulations the scaling rule was found to hold exactly for small and intermediate values of $u_{\infty} R e$ DaIc. For larger values of $u_{\infty} \operatorname{Re}$ DaIc, however, friction in the gap becomes significant as an additional factor influencing the flow. This results in a lowered dimensionless velocity.

The present T-RANS simulations, performed at varied $u_{\infty}, R e$ and $D a$ and fixed Ic and Ig, generally confirm the scaling behavior as found from steady state RANS. The scaled velocity underneath the porous layer is constant within a factor of 2 over a range of over 2 decades variation in the scaling parameter, with again a tendency to decreased velocities at larger values of $u_{\infty} R e$ DaIc. In general, the scaled velocities obtained by T-RANS are somewhat higher than those obtained by steadystate RANS. Unlike the steady RANS simulations, the T-RANS simulations show a deviation from the scaling law for the datapoints at $u_{\infty} R e$ DaIc $=0.02-0.04$, which were obtained at a transitional $R e$ number of 3,900. Because the scaling analysis assumed steady flow, this deviation is therefore not surprising.

In [8], we also presented an engineering correlation for the Nusselt number, averaged over time and angle, for heat transfer to the surface of a solid cylinder covered by a porous layer.

$$
\{\overline{N u}\}=\frac{1}{I g}+\frac{\alpha \cdot(\text { DaIc })^{n} \cdot R e^{n}}{1+\alpha \cdot(\text { DaIc })^{n} \cdot R e^{n}} \cdot\left\{\overline{N u_{b}}\right\}
$$

where, $\alpha=2.93, n=0.82$ and $\left\{\overline{N u_{b}}\right\}$ is the Nusselt number as a function of $R e$ for a bare cylinder. The range of validity of eq. 4 is $\operatorname{Ig} \operatorname{Re}>10^{3}, \operatorname{Ig} \operatorname{Re} \operatorname{DaIc}<10^{-2}$. In Fig. 11 some predicted curves from this correlation for different values of DaIc (all at $I g=0.05)$ are shown and compared to the well-known correlation for the Nusselt number on a bare (i.e. uncovered) cylinder in cross flow. Also shown are averaged Nusselt numbers as predicted by the present T-RANS simulations. Compared to the 
Fig. 11 Nusselt number (averaged over time and angular coordinate) as a function of Reynolds number. Results of current T-RANS simulations are compared to an empirical correlation (4), which was developed based on a two-dimensional RANS study [8]

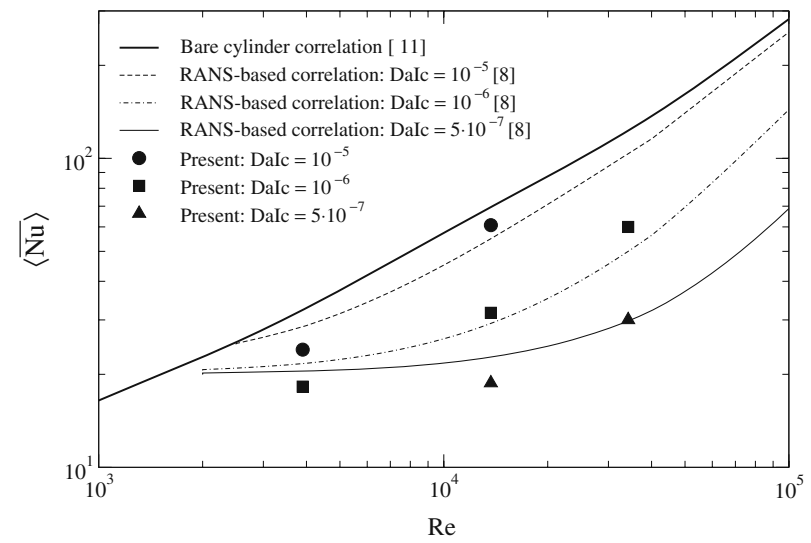

correlation based on steady-state two-dimensional RANS simulations, the T-RANS simulation predict somewhat lower Nusselt numbers at low $R e$ and somewhat higher Nusselt numbers at higher $R e$. This can be explained by two opposing effects as follows:

(1) As shown in Fig. 7 and explained in the previous section, steady-state twodimensional RANS simulations over-predict heat transfer in the back region, due to a erroneous representation of the outwardly directed radial flow in this region. This results in an over-prediction of the average Nusselt number by the RANS approach, on which the empirical correlation is based, when the contribution of this back region to the overall heat transfer is significant, i.e. at low $R e$.

(2) At high values of $R e$, the overall heat transfer is dominated by the heat transfer in the front stagnation point, which depends on the velocity of the air penetrating the porous layer in this point. As shown in Fig. 10 and discussed above, two-dimensional steady state RANS predicts these velocities to be somewhat lower as compared to the velocities predicted by three-dimensional T-RANS. This results in an under-prediction of the average Nusselt number by the RANS approach, on which the empirical correlation is based, when the contribution of the stagnation point to the overall heat transfer is significant, i.e. at high $R e$.

Despite these differences between the earlier RANS simulations and the present, more accurate, T-RANS results, the empirical correlation based on RANS is still found to predict Nusselt numbers within engineering accuracy, i.e. within tens of percents. Also, the overall qualitative behavior of $\mathrm{Nu}$ as a function of $\mathrm{Re}$ and $\mathrm{DaIc}$ is identical in the present T-RANS and earlier RANS simulations.

\section{Conclusions}

In this work we used a T-RANS approach in combination with an RNG $k-\epsilon$ subscale model to study the hydrodynamics and heat/mass transfer for a circular cylinder surrounded at fixed distance by a porous layer in perpendicular cross-flow. 
The results from this approach were validated against our earlier DNS at subcritical $R e=3,900$ [9]. The T-RANS results were also compared to results obtained with two-dimensional steady state RANS simulations with an RNG $k-\epsilon$ model [8].

When comparing the T-RANS results to those from DNS at $R e=3,900$, both for a bare and a covered cylinder, the T-RANS approach was found to be very accurate and obviously significantly cheaper in terms of the computational resources compared to DNS. As in the DNS study, T-RANS showed the flow underneath the porous layer to be periodic, with a frequency locked to that of the vortex shedding at the outer cylinder surface. Both the frequency and amplitude of the transient flow in the fluid gap are well reproduced, with negligible discrepancies compared to DNS.

When comparing the T-RANS results for a covered cylinder at $R e=3,900$ to those from two-dimensional steady state RANS, the T-RANS flow velocities underneath the porous layer were found to be in better agreement with DNS and experimental data than those from RANS, especially in the downstream region. In this region, there is a strong interaction with the vortex shedding in the outer flow. Also for heat/mass transfer the T-RANS approach led to significantly improved results, compared to DNS. This implies that, for the studied geometries and flow ranges, the dynamic flow treatment of T-RANS has significant advantages over steady state RANS.

Keeping in mind that the ultimate goal of computational garment modeling is the simulation of a full-scale, clothed, moving human body in crosswind conditions, it is clear that both DNS and LES approaches are still too computationally expensive, or even not feasible at relevant Reynolds numbers. Steady-state RANS modeling, on the other hand, is unable to capture the precise transient nature of the flow underneath the garment material, leading to inaccurate heat/mass transfer predictions. In between these two extremes, the T-RANS approach as studied in present article is a very promising alternative, combining accurate flow and heat/mass transfer predictions with relatively moderate computational costs. Furthermore, a T-RANS approach as presented here can easily be performed within the context of commercially available general-purpose CFD codes.

Acknowledgements Research on this project is supported and financed by the Prins Maurits Laboratory of the Netherlands Organization for Applied Scientific Research.

Open Access This article is distributed under the terms of the Creative Commons Attribution Noncommercial License which permits any noncommercial use, distribution, and reproduction in any medium, provided the original author(s) and source are credited.

\section{References}

1. Geers, L.F.G.: Developments in modeling NBC protective clothing. Technical Report PML2004A122, TNO-PML, Rijswijk, The Netherlands (2004)

2. Brasser, P., Kaaijk, J.: Modellering bescherming NBC-kleding, 1. Stromingsprofiel tussen kleding en huid. Technical Report PML1998-A107 (in Dutch), TNO-PML, Rijswijk, The Netherlands (1998)

3. Brasser, P.: Modeling the chemical protective performance of NBC clothing material. J. Occup. Env. Hygiene 1, 620-628 (2004)

4. Watanabe, T., Kato, T., Kamata, Y.: The velocity distribution in the inner flow field around a clothed cylinder. SEN-I GAKKAISHI 47, 271-275 (1991) 
5. Kind, R.J., Jenkis, C.A., Broughton, C.A.: Measurement of wind-induced heat transfer through permeable cold-weather clothing. Cold Regions Sci. Technol. 23, 305-316 (1995)

6. Gibson, P.W.: Review of numerical modeling of convection, diffusion, and phase change in textiles. In: Kleijn, C.R., Kawano, S. (eds.) Computational Technologies for Fluid/thermal/ structural/chemical Systems with Industrial Applications, vol. 397-2, pp. 117-126. New York, USA, ASME PVP (1999)

7. Bo, Q., Nakijama, T.: Natural and mixed convection around a cylinder enclosed with porous media-a numerical study on comfort of clothed human being. In: Kleijn, C.R., Kudriavtsev, V. (eds.) Computational Technologies for Fluid/thermal/structural/chemical Systems with Industrial Applications, vol. 424-2, pp. 177-183. New York, USA, ASME PVP (2001)

8. Sobera, M.P., Kleijn, C.R., Brasser, P., van den Akker, H.E.A.: Convective heat and mass transfer to a cylinder sheathed by a porous layer. AIChE J. 49, 3018-3028 (2003)

9. Sobera, M.P., Kleijn, C.R., Van den Akker, H.E.A.: Subcritical flow past a circular cylinder surrounded by a porous layer. Phys. Fluids 18, 038106 (2006)

10. Williamson, C.H.K.: Vortex dynamics in the cylinder wake. Ann. Rev. Fluid Mech. 28, 477-539 (1996)

11. Zdravkovich, M.M.: Flow Around Circular Cylinders. Oxford University Press, Oxford, UK (1997)

12. Hanjalic, K.: Will RANS survive LES? A view of perspectives. J. Fluids Eng. 127, 831-839 (2005)

13. Hanjalic, K.: One point closure models for buoyancy-driven turbulent flows. Ann. Rev. Fluid Mech. 34, 321-347 (2002)

14. Kenjeres, S., Hanjalic, K.: Transient analysis of Rayleigh-Bernard convection with a RANS model. Int. J. Heat Fluid Flow 20, 329-340 (1999)

15. Hanjalic, K., Kenjeres, S.: Reorganization of turbulence structure in magnetic Rayleigh-Benard convection: a T-RANS study. J. Turbul. 1, 1-22 (2000)

16. Hanjalic, K., Kenjeres, S.: T-RANS simulation of deterministic eddy structure in flows driven by thermal buoyancy and lorentz force. Flow Turbul. Combus. 66, 427-451 (2001)

17. Kenjeres, S., Hanjalic, K.: Combined effects of terrain orography and thermal stratication on pollutant dispersion in a town valley: a T-RANS simulation. J. Turbul. 3, 026 (2002)

18. Cullivan, J.C., Williams, R.A., Cross, C.R.: Understanding the hydrocyclone separator through computational fluid dynamics. Chem. Eng. Res. Design 81, 455-466 (2003)

19. Aubin, J., Fletcher, D.F., Xuereb, C.: Modeling turbulent flow in stirred tanks with CFD: the influence of the modeling approach, turbulence model and numerical scheme. Exp. Thermal Fluid Sci. 28, 431-445 (2004)

20. Leonard, B.P.: A stable and accurate convective modelling procedure based on quadratic upstream interpolation. Comp. Meth. Appl. Mech. Eng. 19, 59-98 (1979)

21. Yakhot, V., Orszag, S.A.: Renormalization group analysis of turbulence. J. Sci. Comput. 1, 3-51 (2005)

22. Yakhot, V., Orszag, S.A.: Renormalization-group analysis of turbulence. Phys. Rev. Lett. 57, 1722-1724 (1986)

23. Yakhot, V., Orszag, S.A., Thangam, S., Gatski, T.B., Speziale, C.G.: Development of turbulence models for shear flows by a double expansion technique. Phys. Fluids A 4, 1510-1520 (1992)

24. Velayati, E., Yaghoubi, M.: Numerical study of convective heat transfer from an array of parallel bluff plates. J. Heat Fluid Flow 26, 80-91 (2005)

25. Casey, M., Wintergerste, T.: Quality and Trust in Industrial CFD. ERCOFTAC-Sulzer Innotec, Winterthur, Switzerland (2000)

26. Sobera, M.P., Kleijn, C.R., Brasser, P., van den Akker, H.E.A.: Hydrodynamics of the flow around a circular cylinder sheathed by a porous layer. In: Proceedings of the 2004 ASME Heat Transfer/Fluids Engineering Summer Conference. Charlotte, NC, USA, Published by ASME on CD (2004)

27. Tremblay, F.: Direct and large-eddy simulation of flow around a circular cylinder at subcritical Reynolds numbers. PhD thesis, Munich University of Technology, Germany (2001)

28. Ong, L., Wallace, J.: The velocity field of the turbulent very near wake of a circular cylinder. Exp. Fluids 20, 441-453 (1996)

29. Beaudan, P., Moin, P.: Numerical experiments on the flow past a circular cylinder at sub-critical Reynolds number. Technical Report TF-62, Thermoscience Division, Department of Mechanical Engineering, Stanford University, USA (1994)

30. Breuer, M.: Large eddy simulation of the subcritical flow past a circular cylinder: numerical and modeling aspects. Int. J. Numer. Meth. Fluids 28, 1281-1302 (1998) 
31. Krall, K.M., Eckert, E.R.R.: Local heat transfer around a cylinder at low Reynolds number. J. Heat Trans. 95, 273-277 (1973)

32. Adachi, T., Okamoto, S., Adachi, M.: The effect of sound on the rate of heat transfer from cylinder placed normal to an air stream. Bull. JSME 22, 1407-1415 (1979)

33. Sobera, M.P., Kleijn, C.R., Brasser, P., Van den Akker, H.E.A.: A multi-scale numerical study of the flow, heat and mass transfer in protective clothing. LNCS 3039, 637-644 (2004) 